\title{
Quantum Yield Characterization and Excitation Scheme Optimization of Upconverting Nanoparticles
}

\author{
Liu, Haichun; Xu, Can T.; Jensen, Ole Bjarlin; Andersen, Peter E.; Andersson-Engels, Stefan
}

Published in:

Biomedical Optics 2014

Link to article, DOI:

10.1364/BIOMED.2014.BM3A.54

Publication date:

2014

Document Version

Peer reviewed version

Link back to DTU Orbit

Citation (APA):

Liu, H., Xu, C. T., Jensen, O. B., Andersen, P. E., \& Andersson-Engels, S. (2014). Quantum Yield Characterization and Excitation Scheme Optimization of Upconverting Nanoparticles. In Biomedical Optics 2014 Optical Society of America (OSA). https://doi.org/10.1364/BIOMED.2014.BM3A.54

\section{General rights}

Copyright and moral rights for the publications made accessible in the public portal are retained by the authors and/or other copyright owners and it is a condition of accessing publications that users recognise and abide by the legal requirements associated with these rights.

- Users may download and print one copy of any publication from the public portal for the purpose of private study or research.

- You may not further distribute the material or use it for any profit-making activity or commercial gain

- You may freely distribute the URL identifying the publication in the public portal 


\title{
Quantum yield characterization and excitation scheme optimization of upconverting nanoparticles
}

\author{
Haichun Liu ${ }^{1, *}$, Can T. Xu ${ }^{1}$, Ole B. Jensen ${ }^{2}$, Peter E. Andersen ${ }^{2}$, Stefan \\ Andersson-Engels ${ }^{1}$ \\ ${ }^{1}$ Department of Physics, Lund University, Lund, Sweden \\ ${ }^{2}$ Department of Photonics Engineering, Technical University of Denmark, Roskilde, Denmark \\ *haichun.liu@fysik.lth.se
}

\begin{abstract}
Upconverting nanoparticles suffer from low quantum yield in diffuse optical imaging, especially at low excitation intensities. Here, the power density dependent quantum yield is characterized, and the excitation scheme is optimized based on such characterization.
\end{abstract}

OCIS codes: $160.1435,160.4236,160.5690,190.7220,110.0113$

\section{Background}

Recently, upconverting nanoparticles have drawn an increasing amount of attention as contrast agents for optical bioimaging. They enable autofluorescence-free imaging within the tissue optical window [1], and improved spatial resolution as compared to conventional fluorescence based contrast agents [2]. In addition, they are very photostable [3]. Despite the numerous beneficial properties, a major challenge of the use of UCNPs in biomedical applications, especially in deep tissues, is the power density dependent and relatively low quantum yields (QYs) at the low excitation intensities, required for these applications.

In this study, the QY of $\mathrm{Yb}^{3+}$ sensitized two-photon upconversion (UC) emissions, is characterized, making use of steady state rate equation analysis. Based on such characterization, a pulsed excitation approach is proposed as a superior alternative for pumping UCNPs embedded in deep tissues. The proposed approach allows the utilization of higher intrinsic QY without increasing the side photo-thermal effects compared to the conventional continuous wave (CW) excitation scheme.

\section{Quantum yield characterization of $\mathrm{Yb}^{3+}$ sensitized two-photon upconversion emission}

The mechanism of $\mathrm{Yb}^{3+}$ sensitized two-photon UC emission can be generally depicted by a quasi-three-level model for main UC activators including $\mathrm{Er}^{3+}, \mathrm{Tm}^{3+}$ and $\mathrm{Ho}^{3+}$, as shown in Fig. 1(a). The excitation intensity dependent
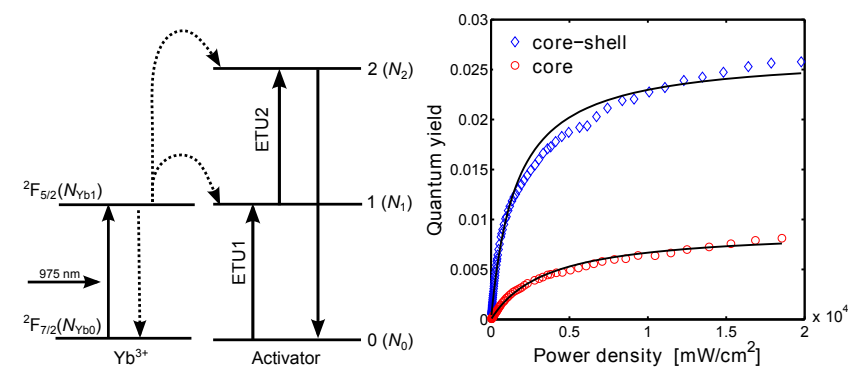

Fig. 1. (a) Schematic energy level diagrams of the $\mathrm{Yb}^{3+}$ and activator ions and the proposed upconversion mechanism following $975 \mathrm{~nm}$ excitation. (b) The QYs of the $800 \mathrm{~nm}$ upconversion emission band of the core $\mathrm{NaYF}_{4}: \mathrm{Yb}^{3+}, \mathrm{Tm}^{3+}$ and core-shell $\mathrm{NaYF}_{4}: \mathrm{Yb}^{3+}, \mathrm{Tm}^{3+} @ \mathrm{NaYF}_{4} \mathrm{UCNPs}^{3}$ The black solid lines stand for the fitted data. 
behavior of the UC emission intensity and QY can be well approximated by the following steady-state rate equations:

$$
\begin{aligned}
\frac{\mathrm{d} N_{\mathrm{Yb} 1}}{\mathrm{~d} t} & =\sigma \rho N_{\mathrm{Yb} 0}-\frac{N_{\mathrm{Yb} 1}}{\tau_{\mathrm{Yb} 1}}=0, \\
\frac{\mathrm{d} N_{1}}{\mathrm{~d} t} & =C_{0} N_{0} N_{\mathrm{Yb} 1}-C_{1} N_{1} N_{\mathrm{Yb} 1}-\frac{N_{1}}{\tau_{1}}=0, \\
\frac{\mathrm{d} N_{2}}{\mathrm{~d} t} & =C_{1} N_{1} N_{\mathrm{Yb} 1}-\frac{N_{2}}{\tau_{2}}=0 .
\end{aligned}
$$

Here, $N_{\mathrm{Yb} 0}$ and $N_{\mathrm{Yb} 1}$ are population densities of states ${ }^{2} \mathrm{~F}_{7 / 2}$ and ${ }^{2} \mathrm{~F}_{5 / 2}$ of $\mathrm{Yb}^{3+}$, respectively, while $N_{0}, N_{1}$ and $N_{2}$ are population densities of states 0,1 and 2 of activator ions, respectively; $\sigma$ is the absorption cross-section of $\mathrm{Yb}^{3+}$ ions; $\rho$ is the excitation photon flux, which is linearly related with power density; $\tau_{1}$ and $\tau_{2}$ are the lifetimes of activator ions at states 1 and 2, respectively, including contributions of radiative and non-radiative relaxation mechanisms, while $\tau_{\mathrm{Yb} 1}$ is the lifetime of $\mathrm{Yb}^{3+}$ ions at ${ }^{2} \mathrm{~F}_{5 / 2}$ state; $C_{0}$ and $C_{1}$ are ETU rates constants characterizing the processes ETU1 and ETU2, respectively. It can be seen from the above set of equations that the QY of the two-photon UC emission can be described by [4],

$$
\eta=\eta_{\mathrm{s}} \cdot \frac{\rho / \rho_{\mathrm{b}}}{1+\rho / \rho_{\mathrm{b}}}
$$

where $\eta_{\mathrm{s}}=C_{0} N_{0} \tau_{\mathrm{Yb} 1} \tau_{2} / \tau_{2}^{\mathrm{rad}}$, and $\rho_{\mathrm{b}}=1 /\left(\tau_{1} \cdot C_{1} \tau_{\mathrm{Yb} 1} \sigma N_{\mathrm{Yb} 0}\right) . \tau_{2}^{\mathrm{rad}}$ is the radiative lifetime of state 2 .

Experimental QY data measured on core $\mathrm{NaYF}_{4}: \mathrm{Yb}^{3+}, \mathrm{Tm}^{3+}$ and core-shell $\mathrm{NaYF}_{4}: \mathrm{Yb}^{3+}, \mathrm{Tm}^{3+} @ \mathrm{NaYF}_{4} \mathrm{UCNPs}^{3}$ can be well fitted through Eq. 2, as shown in Fig. 1(b). The discrepancy could be due to the omission of certain energy transfer processes in the rate equation model and the inaccuracy in the measurement of the excitation power density.

\section{Excitation scheme optimization of upconverting nanoparticles}

As seen in Fig. 1(b), the QY of UCNPs increases with excitation intensity. If a pulsed light source is used to replace an equivalent $\mathrm{CW}$ source with identical average power, upconversion signal gain can be expected, as the pulsed source provides significantly larger excitation intensities in the pulse duration. In addition, as shown, gradual saturation behavior of the QY at high intensities indicates the possible signal gain would vanish if the applied average power is high, because in such a regime larger excitation intensities would not lead to higher QYs [5]. These are confirmed by experimental observations presented in Fig. 2. Here, a pulse source with $2 \mathrm{~Hz}$ repetition rate and a $20 \mathrm{~ms}$ pulse width is compared with a CW source, both providing the same power and power density. As shown, at low power densities, the upconversion signal is increased by almost one order of magnitude through using pulsed excitation, and the signal gain decreases with the applied average power density.

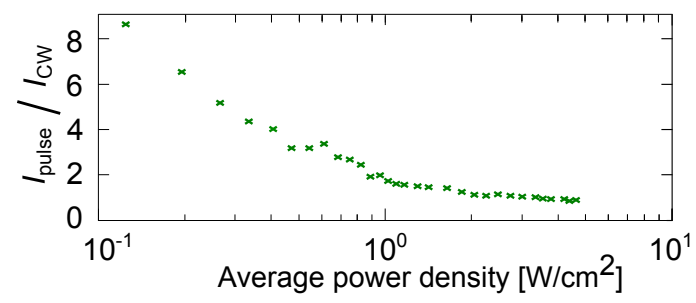

Fig. 2. The NIR upconversignal gain by the pulsed excitation at different average power densities. The pulsed excitation was set to have a $20 \mathrm{~ms}$ pulse width and $2 \mathrm{~Hz}$ repetition rate.

\section{Deep tissue imaging of upconverting nanoparticles using pulsed excitation}

The pulsed excitation constitutes an ideal approach for exciting deeply located UCNPs, since the UC emissions can be enhanced without consuming more excitation energy than an equivalent $\mathrm{CW}$ source, thus not increasing the thermal side-effects of the excitation light. This would in turn lead to higher image quality and larger imaging depth.

The merit of pulsed excitation for diffuse imaging was tested in a 17-mm thick liquid tissue phantom, made of water, intralipid and ink. The phantom was characterized by photon time-of-flight spectroscopy and had a reduced scattering coefficient $\mu_{\mathrm{s}}^{\prime}=10.1 \mathrm{~cm}^{-1}$ and absorption coefficient $\mu_{\mathrm{a}}=0.52 \mathrm{~cm}^{-1}$ at $975 \mathrm{~nm}$. A glass tube with an 
inner diameter of $2 \mathrm{~mm}$, containing the colloidal core-shell UCNPs $(c=1 \mathrm{wt} \%)$, was used as a luminescent inclusion to mimic a UCNP-labeled target. Both a CW excitation, and pulsed excitation scheme at $975 \mathrm{~nm}$ were applied. The average power density impinging on the surface of the tissue phantom was $1.2 \mathrm{~W} / \mathrm{cm}^{2}$ for both excitation approaches. The excitation source and the detector were positioned in a trans-illumination geometry. When buried at a depth of 10 $\mathrm{mm}$ from the source, the luminescent inclusion was barely detectable under CW excitation even with an exposure time of $10 \mathrm{~s}$, as shown in Fig. 3(a). However, by using pulsed excitation, the signal-to-background ratio was significantly increased by a factor of approximately 7 under the same detection conditions, as illustrated in Fig. 3(b). Surprisingly, single-shot imaging of deeply embedded UCNPs is also feasible, benefiting from the high peak power of the single excitation pulse, as shown in Fig. 3(d). The data acquisition time could be significantly shortened without loss in the UC signal quality.

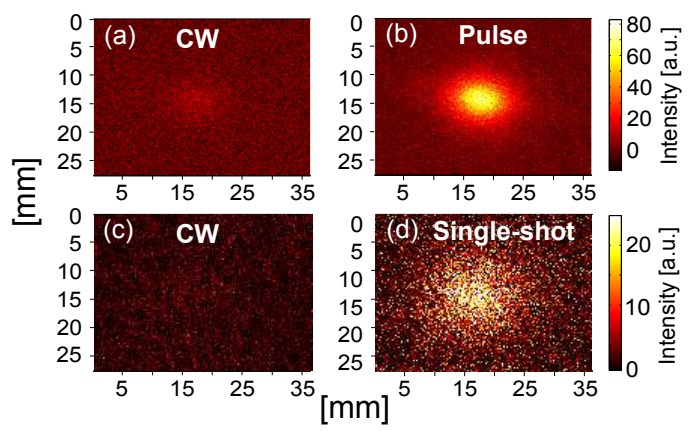

Fig. 3. The NIR UC emission images taken for a luminescent inclusion located at a depth of 10 $\mathrm{mm}$ under (a) $\mathrm{CW}$ and (b) pulsed excitation, detected in a trans-illumination geometry. The average power density was $1.2 \mathrm{~W} / \mathrm{cm}^{2}$, and the exposure time was $10 \mathrm{~s}$ for both excitation approaches. The NIR UC emission images taken for a luminescent inclusion at a depth of $13 \mathrm{~mm}$ under (c) CW and (d) $50 \mathrm{~ms}$ single pulse excitation, detected in a epi-illumination geometry. Both excitation sources provided the maximum permissible power density allowed by ANSI standard for exposure to human skin. The exposure time was $10 \mathrm{~s}$ for the $\mathrm{CW}$ excitation and $1 \mathrm{~s}$ for the single pulse excitation.

\section{Conclusion}

We first characterize theoretically the power density dependent $\mathrm{QYs}$ of $\mathrm{Yb}^{3+}$ sensitized two-photon upconversion emission for arbitrary excitation intensities. Based on such characterization, the pulsed excitation approach is proposed for pumping UCNPs in order to use high intrinsic QYs. The merits of pulsed excitation for diffuse tissue optical imaging is demonstrated. The results suggest that pulsed excitation is a superior excitation scheme to $\mathrm{CW}$ excitation for pump UCNPs in deep tissues.

\section{References}

1. Can T. Xu, Niclas Svensson, Johan Axelsson, Pontus Svenmarker, Gabriel Somesfalean, Guanying Chen, Huijuan Liang, Haichun Liu, Zhiguo Zhang, and Stefan Andersson-Engels. Autofluorescence insensitive imaging using upconverting nanocrystals in scattering media. Applied Physics Letters, 93:171103, 2008.

2. Can T. Xu, Johan Axelsson, and Stefan Andersson-Engels. Fluorescence diffuse optical tomography using upconverting nanoparticles. Applied Physics Letters, 94:251107, 2009.

3. Shiwei Wu, Gang Han, Delia J. Milliron, Shaul Aloni, Virginia Altoe, Dmitri V. Talapin, Bruce E. Cohen, and P. James Schuck. Non-blinking and photostable upconverted luminescence from single lanthanide-doped nanocrystals. Proceedings of the National Academy of Sciences, 106:10917, 2009.

4. Haichun Liu, Can T. Xu, David Lindgren, Haiyan Xie, Diana Thomas, Carsten Gundlach, and Stefan AnderssonEngels. Balancing power density based quantum yield characterization of upconverting nanoparticles for arbitrary excitation intensities. Nanoscale, 5:4770, 2013.

5. Haichun Liu, Can T. Xu, Gokhan Dumlupinar, Ole Bjarlin Jensen, Peter E. Andersen, and Stefan AnderssonEngels. Deep tissue optical imaging of upconverting nanoparticles enabled by exploiting higher intrinsic quantum yield through using millisecond single pulse excitation with high peak power. Nanoscale, 5:10034, 2013. 